\title{
Relationships Between Several Quadrat-Based Statistical Measures Used to Characterize Spatial Aspects of Disease Incidence Data
}

\author{
M. S. Ridout and X.-M. Xu
}

Horticulture Research International, East Malling, West Malling, Kent, ME19 6BJ, United Kingdom. Accepted for publication 8 March 2000.

\section{ABSTRACT}

Ridout, M. S., and Xu, X.-M. 2000. Relationships between several quadratbased statistical measures used to characterize spatial aspects of disease incidence data. Phytopathology 90:568-575.

This article investigates the relationships between various statistical measures that are used to summarize spatial aspects of disease incidence data. The focus is on quadrat data in which each plant in a quadrat is classified as diseased or healthy. We show that spatial autocorrelation plays a central role via the mean intraclass correlation, $\rho$, which is defined as the average correlation of the disease status of all pairs of plants within the quadrat. The value of $\rho$ determines the variance of the number of infected plants in the quadrat and, if this variable follows a beta-binomial distribution, the heterogeneity parameter of the beta-binomial distribution is directly related to the mean intraclass correlation. We consider in detail a model in which the spatial autocorrelation depends only on the distance between the plants. For illustration, we consider a specific autocorrelation model that was derived from simulated data. We show that this model leads, approximately, to the binary form of the power law relating the variance of the number of infected plants per quadrat to the mean. Using an approximation technique, we then show how the index of dispersion is related to quadrat size and shape. The index of dispersion increases with quadrat size. The rate of increase is dependent on quadrat shape, but the effect of quadrat shape is small in comparison to the effect of quadrat size. Finally, we note that if the spatial autocorrelation depends on the relative orientation of the plants, as well as the distance between them, there are connections with distance class methods.
Recently, there has been considerable interest in statistical aspects of disease incidence data, in which individual plants or plant parts are classified as diseased or healthy $(10-13,15,25,26)$. The individuals often occur in groups that may arise naturally (e.g., leaves on a shoot) or artificially (e.g., plants in a quadrat). In this paper, we limit our attention to the incidence of disease on whole plants grown in a rectangular grid and consider quadrat data in which each plant in the quadrat is classified as diseased or healthy.

Many different methods have been used to characterize spatial aggregation for disease incidence data. These include the fitting of distributions, such as the beta-binomial distribution, to quadrat data $(11,15)$ and the study of variance-mean relationships for such data $(10,15)$, the use of spatial autocorrelation techniques $(2,7,15)$, geostatistical methods $(3,8,21)$, and distance class methods and related approaches $(5-7,9,18,19)$.

The aim of this paper is to demonstrate some relationships that exist between these different approaches, extending the work of Madden and Hughes $(10,11)$, who have shown links between the variance, the intraclass correlation, and the dispersion parameter of the beta-binomial distribution. We begin by considering the interpretation of correlation between two variables when these variables are binary. We then show that the variance of the number of infected plants per quadrat depends on the average correlation of the disease status of all pairs of plants within the quadrat. This is determined by the spatial autocorrelation. For a specific autocorrelation model, based on simulated data (25), in which the correlation depends only on the distance between the plants, we show that the variance of the number of infected plants per quadrat follows, approximately, the binary form of the power law (10). Using a numerical approximation technique, we then investigate how the

Corresponding author: X.-M. Xu; E-mail address: xiangming.xu@hri.ac.uk

Publication no. P-2000-0405-010

(C) 2000 The American Phytopathological Society size and shape of the quadrat affect the index of dispersion (the true variance divided by the variance that would be expected if the number of infected plants followed a binomial distribution).

Finally, we note that if the correlation of the disease status of two plants depends on their relative orientation, as well as the distance between them, there are connections with distance class methods (6).

\section{SOME BASIC PROPERTIES OF BINARY RANDOM VARIABLES}

Disease incidence is an example of a binary variable, one that can take only two possible values. In this section, we describe some basic properties of binary random variables.

Distribution of a binary random variable. Let $X$ denote the disease assessment of a particular plant, in which

$$
X= \begin{cases}0 & \text { if the plant is healthy } \\ 1 & \text { if the plant is diseased }\end{cases}
$$

and let $\pi$ denote the probability that the plant is diseased, $\operatorname{Pr}(X=$ $1)=\pi$. Thus, $\operatorname{Pr}(X=0)=1-\pi$, and the value of $\pi$ determines the distribution of $X$ completely. In particular, the mean and variance are given by $E(X)=\pi$ and $\operatorname{var}(X)=\pi(1-\pi)$. This distribution, known as the Bernoulli distribution, is the only possible probability distribution for a binary random variable.

Correlation between binary variables. The following results are well known in statistical literature (17) and have appeared also in plant pathology literature (15).

For continuous random variables, correlation provides a measure of linear association between two variables. For binary variables, this interpretation of correlation is not very helpful, because a pair of binary variables can have only four distinct values. Nonetheless, the correlation between two binary variables, $X_{1}$ and $X_{2}$, may be formally defined in the same way as for any other pair of random variables, namely 


$$
\operatorname{corr}\left(X_{1}, X_{2}\right)=\frac{\operatorname{cov}\left(X_{1} X_{2}\right)}{\sqrt{\operatorname{var}\left(X_{1}\right) \operatorname{var}\left(X_{2}\right)}}=\frac{E\left(X_{1} X_{2}\right)-E\left(X_{1}\right) E\left(X_{2}\right)}{\sqrt{\operatorname{var}\left(X_{1}\right) \operatorname{var}\left(X_{2}\right)}}
$$

We shall assume that, when considered individually, $X_{1}$ and $X_{2}$ have the same Bernoulli distribution (the probability of being diseased is the same for both plants, this being the overall probability of disease for the population of interest). Equation 1, therefore, simplifies to

$$
\operatorname{corr}\left(X_{1}, X_{2}\right)=\frac{E\left(X_{1} X_{2}\right)-\pi^{2}}{\pi(1-\pi)}
$$

Moreover, for binary variables, $E\left(X_{1} X_{2}\right)=\operatorname{Pr}\left(X_{1}=1, X_{2}=1\right)$, and we can, therefore, rearrange equation 2 to give

$$
\operatorname{Pr}\left(X_{1}=1, X_{2}=1\right)=\pi^{2}+\pi(1-\pi) \delta
$$

in which $\delta=\operatorname{corr}\left(X_{1}, X_{2}\right)$. Using the fact that $\operatorname{Pr}\left(X_{1}=1\right)=\operatorname{Pr}\left(X_{2}=\right.$ $1)=\pi$, we can similarly calculate that

$$
\operatorname{Pr}\left(X_{1}=0, X_{2}=0\right)=(1-\pi)^{2}+\pi(1-\pi) \delta
$$

and

$$
\operatorname{Pr}\left(X_{1}=0, X_{2}=1\right)=\operatorname{Pr}\left(X_{1}=1, X_{2}=0\right)=\pi(1-\pi)(1-\delta)
$$

$(15,17)$. These joint probabilities provide an interpretation of correlation for binary variables. For a given value of $\pi$, a positive correlation increases the probability that both plants will be diseased or both will be healthy, in comparison to the probabilities that arise when the variables are uncorrelated $(\delta=0)$. Conversely, the probability that one plant will be diseased and the other healthy is reduced. Indeed, the probability that both plants will have the same response is

$$
1-2 \pi(1-\pi)(1-\delta)
$$

$(15,17)$. Negative correlations are possible in principle, but seldom arise in practice. Negative values of $\delta$ must satisfy the inequality $\delta \geq \max [-\pi /(1-\pi),-(1-\pi) / \pi]$ to ensure that the joint probabilities are nonnegative.

\section{QUADRAT DATA}

Variance at a particular incidence of disease. We consider a rectangular array of plants in which the within-row spacing is $w$ and the between-row spacing is $b$. Suppose that a rectangular quadrat spanning $r$ rows and $c$ columns is sampled, and let $n=r c$ denote the total number of plants in the quadrat. A binary random variable $X_{i}$ indicates the disease status of the $i$ th plant in the quadrat $(i=1, \ldots, n)$. The total number of diseased plants in the quadrat is, therefore, $Y=\Sigma_{i} X_{i}$.

If $\pi$ denotes the probability that a randomly selected plant from the population is infected, then $E\left(X_{i}\right)=\pi$ and, hence, $E(Y)=n \pi$. This follows from the fact that the expectation of a sum of random variables is the sum of their individual expectations. We now investigate the variance of $Y$. This is complicated by the fact that the disease status of pairs of plants may be correlated. Using the standard formula for the variance of a sum of random variables

$$
\operatorname{var}(Y)=\sum_{i=1}^{n} \operatorname{var}\left(X_{i}\right)+2 \sum_{i<j} \operatorname{cov}\left(X_{i}, X_{j}\right)
$$

and, since $\operatorname{var}\left(X_{i}\right)=\pi(1-\pi)$, this can be equivalently written as

$$
\operatorname{var}(Y)=n \pi(1-\pi)+2 \pi(1-\pi) \sum_{i<j} \operatorname{corr}\left(X_{i}, X_{j}\right)
$$

We can also write this equation as

$$
\operatorname{var}(Y)=n \pi(1-\pi)[1+(n-1) \rho]
$$

in which

$$
\rho=\frac{1}{n(n-1) / 2} \sum_{i<j} \operatorname{corr}\left(X_{i}, X_{j}\right)
$$

If infection occurs at random, the binary variables will be uncorrelated, giving $\rho=0$ and $\operatorname{var}(Y)=n \pi(1-\pi)$, and the distribution of $Y$ is binomial (15). It may be shown that the average correlation must satisfy the inequality

$$
\rho \geq \frac{-1}{n-1}+\frac{\omega(1-\omega)}{n(n-1) \pi(1-\pi)}
$$

in which $\omega=n \pi-\operatorname{INT}(n \pi)$ and $\operatorname{INT}(x)$ denotes the integer part of $x$ (20). Except when $\pi=1 / 2$, this is a more stringent constraint than that for continuous random variables, in which the constraint is $\rho \geq$ $-1 /(n-1)$.

Equation 3 is a standard form for the variance of a grouped binary variable such as $Y(20)$ and may be derived in two ways. First, we consider the possibility that the incidence of disease varies from quadrat to quadrat in a random way, due, for example, to spatial variability in environmental factors. This gives rise to a mixture distribution for $Y$. The binomial parameter $\pi$ is replaced by a random variable $P$, with $E(P)=\pi$ and $\operatorname{var}(P)=\rho \pi(1-\pi)$. Conditional on the mixing parameter $P$, the distribution is binomial, but it can be shown that the unconditional variance of $Y$ is given by equation 3. Specific distributions for $Y$ may be derived by specifying the distribution of $P$. Commonly, $P$ is assumed to follow a beta distribution, as this gives a flexible range of distributions for $P$ and, hence, for $Y(11,15)$.

The second derivation of equation 3 assumes that all pairs of binary variables $\left(X_{i}, X_{j}\right)$ have equal correlation $\rho$, which is termed the intraclass or intracluster correlation. This is sometimes called the common correlation model (20). The assumption of equal correlation is not usually realistic when the binary variables have a spatial structure, because correlation, which is then spatial autocorrelation, often declines with distance. However, we have shown that equation 3 continues to apply, if $\rho$ is defined as the mean of the complete set of pairwise correlations (equation 4). Since $\rho$ is a correlation, $\rho \leq 1$, and this implies that $\operatorname{var}(Y) \leq n^{2} \pi(1-\pi)$, a result that was derived by a different method by Yang (26).

In terms of spatial pattern, positive values of $\rho$ imply spatial aggregation or clustering, whereas $\rho=0$ implies a random pattern of disease incidence. A negative value of $\rho$ would imply some sort of regularity in the pattern of disease, whereby the presence of a diseased plant reduced the likelihood of neighboring plants being diseased. This explains why negative values of $\rho$ seldom occur in practice. Negative values of $\rho$ cannot arise in the mixture derivation of equation 3 (4).

Variance as a function of disease incidence. Hughes and Madden $(10,15)$ have presented a binary power law relating the variance of disease incidence to mean incidence, with the general form

$$
\operatorname{var}(Y)=A \pi^{b}(1-\pi)^{c}
$$

Equation 5 might be used, for example, to model the variancemean relationship at different times during the development of an epidemic or to compare data from different experimental treatments. It is analogous to Taylor's power law for count data (23) and has been shown to provide a good fit to many data sets (15). Frequently, the simpler two-parameter model with $c=b$ is adequate. This simpler model implies that the variance is greatest when $\pi=1 / 2$; more generally, the maximum variance occurs when $\pi=b /(b+c)$, provided $b$ and $c$ are positive.

The effects of factors such as the spatial distribution of initial infections and the spore dispersal distribution on the variance-mean relationship for disease incidence data have been investigated in simulation models $(25,26)$. It is clear from equations 3 and 4 that $\operatorname{var}(Y)$ can be determined for any value of $\pi$, at least in principle, provided that we know how $\operatorname{corr}\left(X_{i}, X_{j}\right)$ varies with $\pi$. For the moment, we shall make the simplifying assumption that $\operatorname{corr}\left(X_{i}, X_{j}\right)$ depends only on the distance between the two plants, $d_{i j}$. We can, therefore, write

$$
\operatorname{corr}\left(X_{i}, X_{j}\right)=\gamma\left(d_{i j}, \pi\right)
$$


Xu and Ridout (25) found the following spatial autocorrelation model to be useful in describing data from computer-simulated epidemics

$$
\gamma\left(d_{i j}, \pi\right)=S \pi^{u}(1-\pi)^{v} \exp \left[-t d_{i j}(1-\pi)\right]
$$

in which $S$ is a scaling parameter, $u$ and $v$ are power law parameters, and $t$ determines the rate of decrease of correlation with distance, at fixed incidence. We shall use this specific autocorrelation model for illustration in the development that follows, though we could equally have explored alternative models.
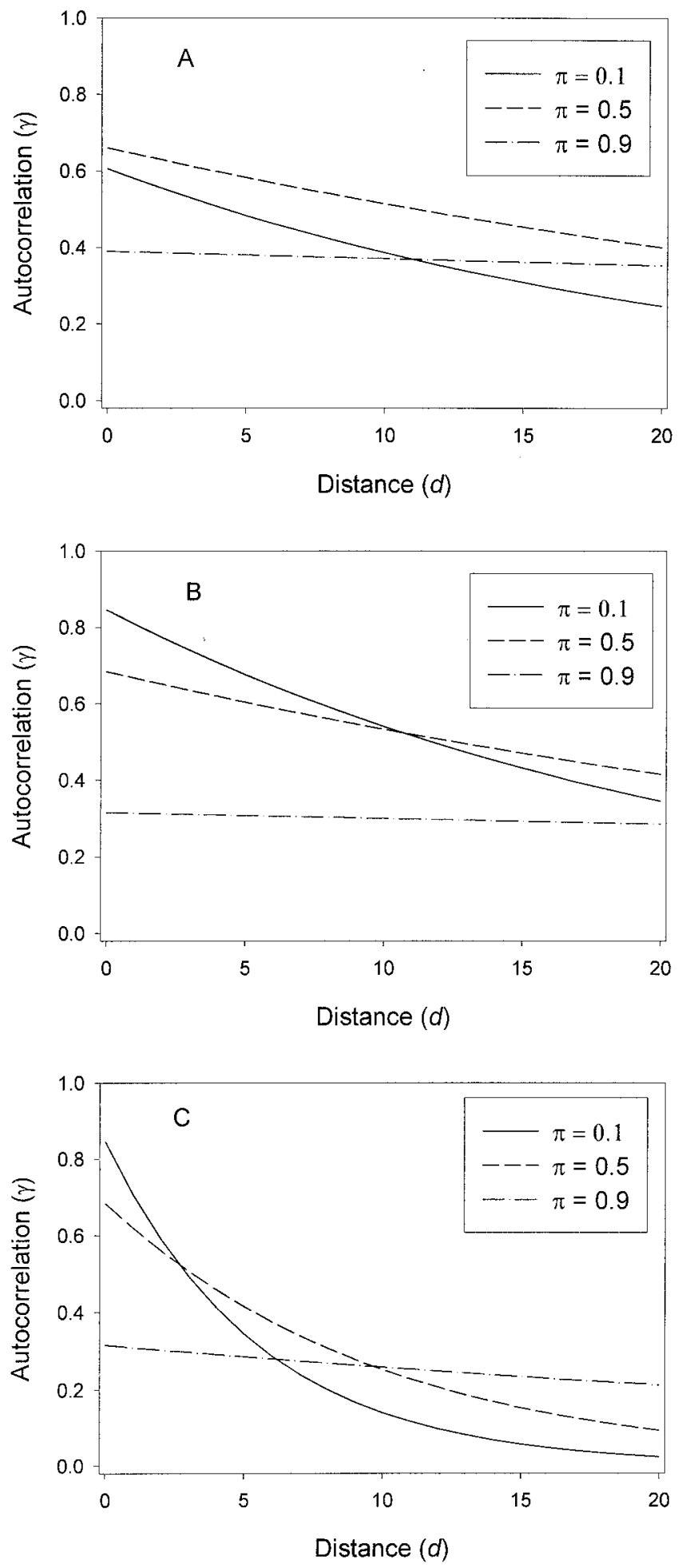

Fig. 1. Autocorrelation as a function of distance for different values of disease incidence, $\pi$, based on equation 7. Parameter values were $\mathbf{A}, t=0.05, u=0.2$, and $v=0.4 ; \mathbf{B}, t=0.05, u=0.05$, and $v=0.5$; and $\mathbf{C}, t=0.2, u=0.05$, and $v=$ 0.5. A to $\mathbf{C}, S=1$.
Figure 1 shows autocorrelation as a function of distance, based on equation 7 , for three levels of disease incidence $(\pi=0.1,0.5$, and 0.9). The values of the parameters in Figure $1 \mathrm{~A}$ are $S=1, t=$ $0.05, u=0.2$, and $v=0.4$, typical of those obtained from the simulation results in Table 3 of Xu and Ridout (25). In Figure 1B, the ratio of the power law parameters, $v / u$, is greatly increased by taking $u=0.05$ and $v=0.5$. This has little effect on the autocorrelation at $\pi=0.5$, but increases autocorrelation at $\pi=0.1$ and decreases it slightly at $\pi=0.9$. In Figure $1 \mathrm{C}$, the parameter values are $S=1, t=0.2, u=0.05$, and $v=0.5$, so that $t$ is four times greater than that in Figure 1B, resulting in a much more rapid decline of autocorrelation with distance. Because the exponential component of equation 7 includes the term $1-\pi$, autocorrelation declines more slowly with distance as $\pi$ increases. In particular, the decline is extremely gradual when $\pi=0.9$.

$\mathrm{Xu}$ and Ridout (25) also calculated intraclass correlation, $\rho_{5,5}(\pi)$, for a quadrat size of $5 \times 5$ and proposed the following power law model.

$$
\rho_{5,5}(\pi)=E \pi^{f}(1-\pi)^{g}
$$

In fact, if we assume that equation 7 correctly describes spatial autocorrelation, $\rho_{5,5}(\pi)$ is determined by substituting equation 7 into equation 4 to give

$$
\rho_{5,5}(\pi)=\frac{2}{25 \times 24} S \pi^{u}(1-\pi)^{v} \sum_{i<j} \exp \left[-t d_{i j}(1-\pi)\right]
$$

Equation 9 is not exactly equivalent to equation 8 . Nonetheless, equation 8 may provide a very good approximation to equation 9 . The variance of $Y$, which we now write as $V_{5,5}(\pi)$ to emphasize the dependence on $\pi$ and on the dimensions of the quadrat, may be obtained by substituting the value of $\rho_{5,5}(\pi)$ from equation 9 into equation 3. Again, this will not yield an exact power law relationship, but equation 5 may nonetheless provide a very good approximation.

We illustrate these approximations in Figure 2. Figure 2A shows the exact value of $\rho_{5,5}(\pi)$ from equation 9 together with an approximation using equation 8 with $E=0.936, f=0.219$, and $g=$ 0.381 . The values of the parameters $S, t, u$, and $v$ were those used for Figure 1A. The two curves are practically indistinguishable. Figure $2 \mathrm{~B}$ shows the exact variance $V_{5,5}(\pi)$ and an approximation based on equation 5 with $A=569, b=1.19$, and $c=1.34$. Again, the two curves are essentially indistinguishable. Figure $2 \mathrm{~B}$ also shows a symmetrical approximation with $b=c=1.23$. This is also similar to the exact $V_{5,5}(\pi)$, and extensive data would be required to distinguish between the two curves experimentally.

Figure 2C and D shows the corresponding graphs based on the parameter values used in Figure 1B. The large value of the ratio $v / u$ results in a highly asymmetrical function, $\rho_{5,5}(\pi)$. Both $\rho_{5,5}(\pi)$ and $V_{5,5}(\pi)$ are approximated well by power law relationships. The approximation shown for $\rho_{5,5}(\pi)$ is based on $E=0.936, f=0.069$, and $g=0.481$. The variance function $V_{5,5}(\pi)$ is less symmetrical about $\pi=0.5$ than the equivalent function in Figure $2 \mathrm{~B}$, but is much more symmetrical than $\rho_{5,5}(\pi)$. The asymmetrical power law approximation to $V_{5,5}(\pi)$ is based on $A=557, b=1.05$, and $c=$ 1.41 , and the symmetrical approximation uses $b=c=1.23$.

Figure $2 \mathrm{E}$ and $\mathrm{F}$ shows results when the parameters in equation 7 are as in Figure 1C. The larger value of $t$ gives more symmetrical results than those in Figure $2 \mathrm{C}$ and D. Once again, $\rho_{5,5}(\pi)$ and $V_{5,5}(\pi)$ are approximated well by power law relationships. The approximation shown for $\rho_{5,5}(\pi)$ is based on $E=0.721, f=0.109$, and $g=0.351$. The asymmetrical power law approximation to $V_{5,5}(\pi)$ is based on $A=464, b=1.10$, and $c=1.36$, and the symmetrical approximation again has $b=c=1.23$.

The approximate values of the parameters $A, b$, and $c$, and $E, f$, and $g$ for Figure 2 were determined using an interactive computer program, to give good visual agreement. This informal procedure is adequate here because our objective is simply to establish that good approximations may be obtained, rather than to determine 'optimal' approximations. 
We have shown, therefore, that in several numerical examples the spatial autocorrelation model given by equation 7 implies, to an extremely good approximation, the power law models for intraclass correlation (equation 8) and variance (equation 5). In all examples, the variance function $V_{5,5}(\pi)$ was more symmetrical about $\pi=0.5$ than the intraclass correlation function $\rho(\pi)$, and it can be shown mathematically that this will always be the case when the intra- class correlation has the power law form of equation 8 (M. S. Ridout, unpublished data).

If equation 8 holds, then substitution into equation 3 gives, after some rearrangement,

$$
\operatorname{var}(Y)=n(n-1) E \pi^{f+1}(1-\pi)^{g+1}+n \pi(1-\pi)
$$

Particularly if $n$ is large, the second term in this expression will
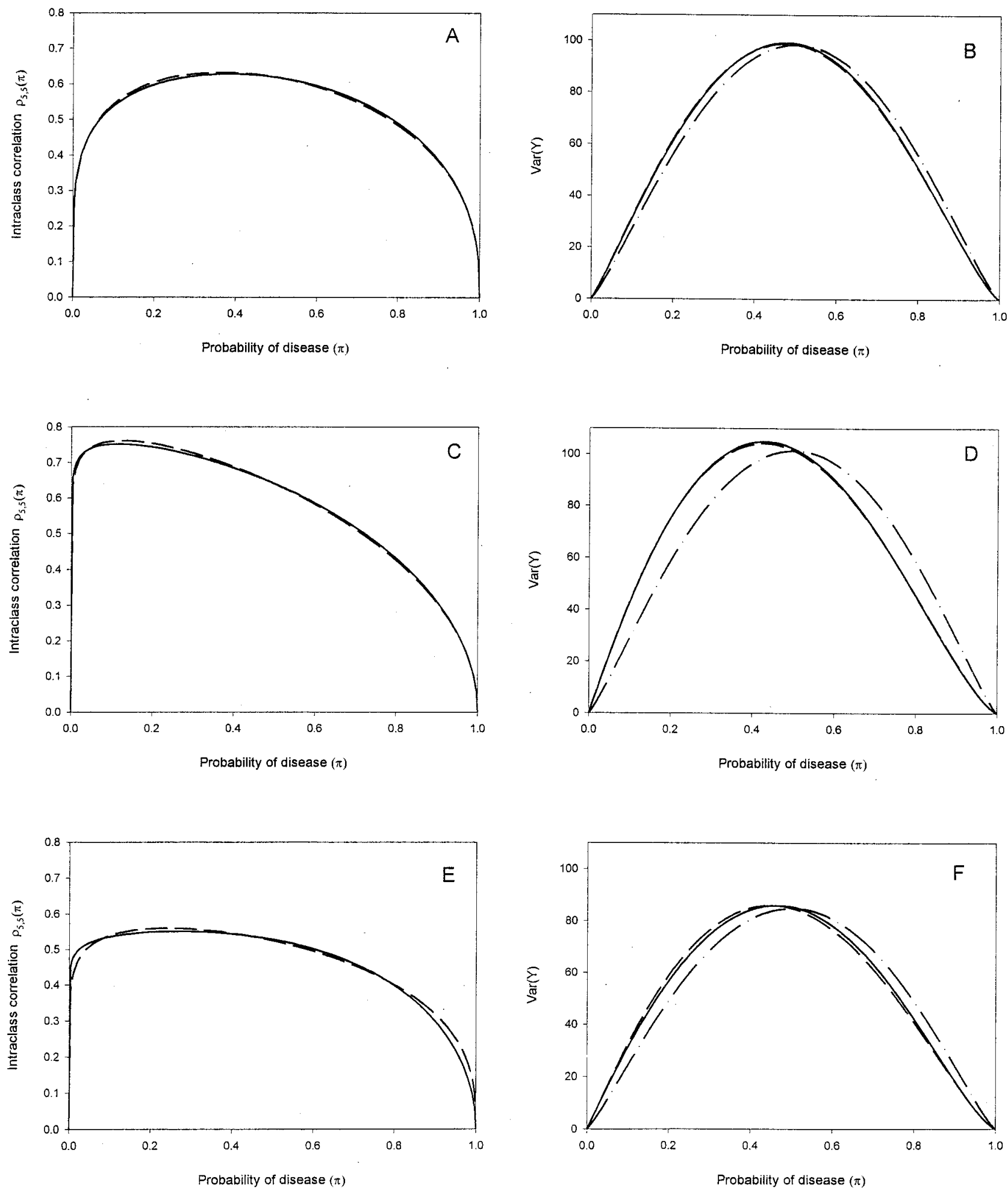

Fig. 2. A, C, and $\mathbf{E}$, Intraclass correlation and $\mathbf{B}, \mathbf{D}$, and $\mathbf{F}$, variance for a $5 \times 5$ quadrat as functions of disease incidence, $\pi$. The solid lines indicate the true relationships based on the autocorrelation model given by equation 7 . The dotted-dashed lines are approximations using an asymmetrical power law. B, D, and $\mathbf{F}$, Dashed lines are symmetrical power law approximations. The parameter values in equation 7 were $\mathbf{A}$ and $\mathbf{B}, t=0.05, u=0.2$, and $v=0.4 ; \mathbf{C}$ and $\mathbf{D}, t=0.05$, $u=0.05$, and $v=0.5$; and $\mathbf{E}$ and $\mathbf{F}, t=0.2, u=0.05$, and $v=0.5$. A to $\mathbf{F}, S=1$. 
often be negligible in comparison to the first term. Thus, the power law model for intraclass correlation implies, approximately, the power law model for variance. The power coefficients are increased by 1 and the scaling parameter is multiplied by a factor of $n(n-1)$. These approximate relationships are seen to hold for the parameter values used in Figure 2.

Approximating the mean pairwise correlation. The previous section has shown the key role played by equation 4 in linking spatial autocorrelation, intraclass correlation, and variance. Evaluation of the right-hand side of equation 4 requires averaging the autocorrelation between all $n(n-1) / 2$ pairs of plants within the quadrat. As far as we are aware, there is no simple formula for the mean correlation and it must be evaluated numerically. The calculations can be done very rapidly by computer, particularly if the pairwise correlation is a function of distance only. Nonetheless, it is useful in some circumstances to have an approximate mathematical formula for the mean correlation, and we now outline a method of deriving such a formula.

For a particular value of $\pi$, we may rewrite equation 4 as

$$
\rho(\pi)=E[\gamma(D)]
$$

in which we have suppressed the dependence of $\gamma$ on $\pi$, and in which $D$ is a random variable denoting the distance between two distinct and randomly chosen plants within the quadrat. A standard technique for approximating the expectation of the function $f(Z)$ of the random variable $Z$, based on a Taylor series expansion of the function, uses the following formula (14).

$$
E[f(Z)] \approx f[E(Z)]+\frac{1}{2} f^{\prime \prime}[E(Z)] \operatorname{var}(Z)
$$

in which $E[$.$] denotes expectation and f^{\prime \prime}[$.$] is the second deriva-$ tive of the function $f[$.].

This formula is not immediately helpful here because there is no simple expression for $E(D)$. However, the mean and variance of $D^{2}$ are

$$
E\left(D^{2}\right)=\frac{r c\left[w^{2}\left(c^{2}-1\right)+b^{2}\left(r^{2}-1\right)\right]}{6(r c-1)}
$$

$$
\begin{aligned}
& \operatorname{var}\left(D^{2}\right)= \\
& \frac{r c\left\{b^{4}\left(r^{2}-1\right)\left[r c\left(7 r^{2}-13\right)-12 r^{2}+18\right]+w^{4}\left(c^{2}-1\right)\left[r c\left(7 c^{2}-13\right)-12 c^{2}+18\right]-10 b^{2} w^{2}\left(c^{2}-1\right)\left(r^{2}-1\right)\right\}}{180(r c-1)^{2}}
\end{aligned}
$$

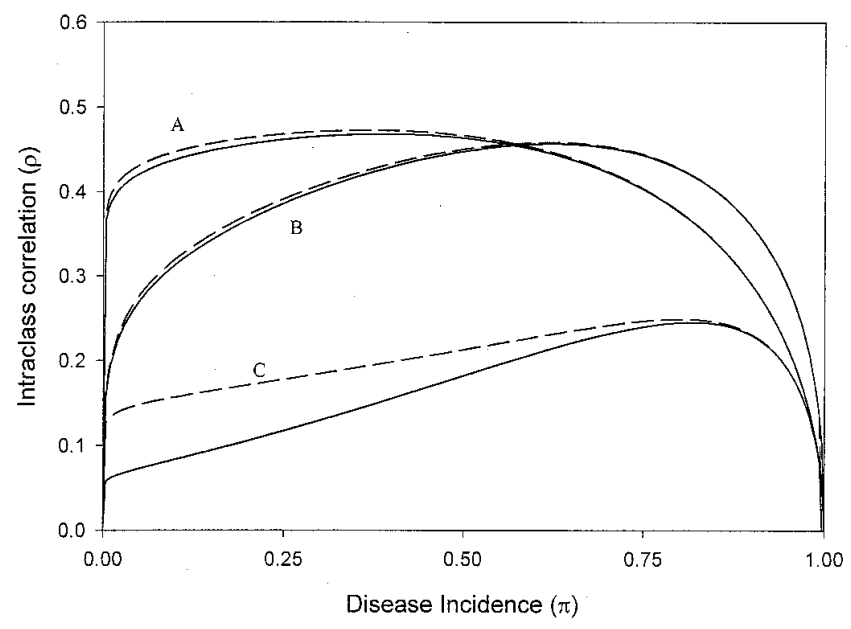

Fig. 3. Exact (solid line) and approximate (dashed line) values of intraclass correlation in a $50 \times 2$ quadrat as a function of disease incidence, $\pi$. Parameter values were $\mathbf{A}, t=0.05, u=0.2$, and $v=0.4 ; \mathbf{B}, t=0.05, u=0.05$, and $v=$ 0.5 ; and $\mathbf{C}, t=0.2, u=0.05$, and $v=0.5$. A to $\mathbf{C}, S=1$.
Derivation of these formulas involves the use of standard results on sums of powers of consecutive integers. We may, therefore, apply equation 10 with $Z=D^{2}$ and $f(Z)=\gamma(\sqrt{Z})$.

To illustrate this method, we take

$$
\gamma(D)=\exp [-t D(1-\pi)]
$$

(compare it with equation 7), which implies

$$
f(Z)=\exp [-t(1-\pi) \sqrt{Z}]
$$

and, therefore,

$$
f^{\prime \prime}(Z)=\frac{t\{(1-\pi)[1+t(1-\pi) \sqrt{Z}]\}}{4 Z^{3 / 2}} \exp [-t(1-\pi) \sqrt{Z}]
$$

Equations 11 to 14 may then be substituted into equation 10 , with $Z=D^{2}$, to give an approximate formula for the mean intraclass correlation $\rho(\pi)$ as a function of the number of rows $(r)$ and columns $(c)$ in the quadrat and of the between- $(b)$ and within- $(w)$ row spacings. Typically, the approximate formula for $\rho(\pi)$ is very lengthy and does not lead to any immediate theoretical insight. However, it is easily investigated graphically.

For a $5 \times 5$ quadrat, with $b=w=1$, and using the same values of the parameters $S, t, u$, and $v$ as in Figure 1, the approximations to $\rho(\pi)$ are extremely accurate for all values of $\pi$. To show the limitations of the approximation more clearly, we consider instead a $50 \times 2$ quadrat. The maximum interplant distance within this quadrat is more than seven times greater than that in the $5 \times 5$ quadrat. Figure 3 shows the true value of $\rho(\pi)$ and the corresponding approximation for each of the three sets of parameter values considered in Figure 1. The approximation is very accurate, except when $t=0.2$, though even here the approximation improves as $\pi$ increases. This is because the adequacy of the approximation is related to the degree of curvature in the autocorrelation function, which, as we have seen in Figure 1, decreases as $\pi$ increases.

Effects of quadrat size and shape on the index of dispersion. For grouped binary data, the index of dispersion, $I(\pi)$, is defined as the variance of the number of infected plants per quadrat divided by the theoretical variance for a binomial distribution (15). From equation 3

$$
I(\pi)=1+(n-1) \rho(\pi)
$$

in which $n=r c$. The approximation to $\rho(\pi)$ derived in the previous section leads to an approximation to $I(\pi)$. Figure 4 plots this approximation as a function of quadrat area based on the autocorrelation function given by equation 7 . Figure $4 \mathrm{~A}, \mathrm{C}$, and $\mathrm{E}$ is based on $S=1, t=0.05, u=0.05$, and $v=0.5$, while Figure $4 \mathrm{~B}, \mathrm{D}$, and $\mathrm{F}$ used $t=0.2$. These are two of the three sets of values used earlier to investigate the functions $\rho_{5,5}(\pi)$ and $V_{5,5}(\pi)$. Different values of $\pi$ were used, as shown on the individual graphs in Figure 4. The approximations were based on $b=w=1$, to coincide with the simulations upon which the autocorrelation model is based (25). Each graph in Figure 4 shows three separate lines. These correspond to different shapes of quadrat, in which the ratio $r / c$ was $1: 1,5: 1$, or $10: 1$.

For $t=0.05$, the index of dispersion increases approximately linearly with increasing quadrat area $(=r c)$ over this range of quadrat sizes. This is because the decline in autocorrelation with distance is approximately linear (Fig. 1B). The rate of increase varies with $\pi$. The index of dispersion is greatest for square quadrats and becomes progressively smaller as the quadrats become elongated. However, the effect of quadrat shape is generally quite small in comparison to the effect of quadrat size.

For $t=0.2$, the decline in autocorrelation with distance shows more curvature (Fig. 1C), and this leads to greater curvature in the relationship between the index of dispersion and the quadrat area and a greater effect of quadrat shape. 
These conclusions are based on the approximate value of $I(\pi)$. However, as discussed earlier, the approximation is generally quite accurate over the range of quadrat sizes considered. Consider, for example, a $30 \times 3$ quadrat. For $t=0.05$, the exact values of $I(0.1)$, $I(0.5)$, and $I(0.9)$, based on an equation analogous to equation 9, are 50.7, 48.7, and 27.6, respectively. The corresponding approximate values are 50.4, 48.7, and 27.6, respectively. For $t=0.2$, the exact values of $I(0.1), I(0.5)$, and $I(0.9)$ are 22.1,
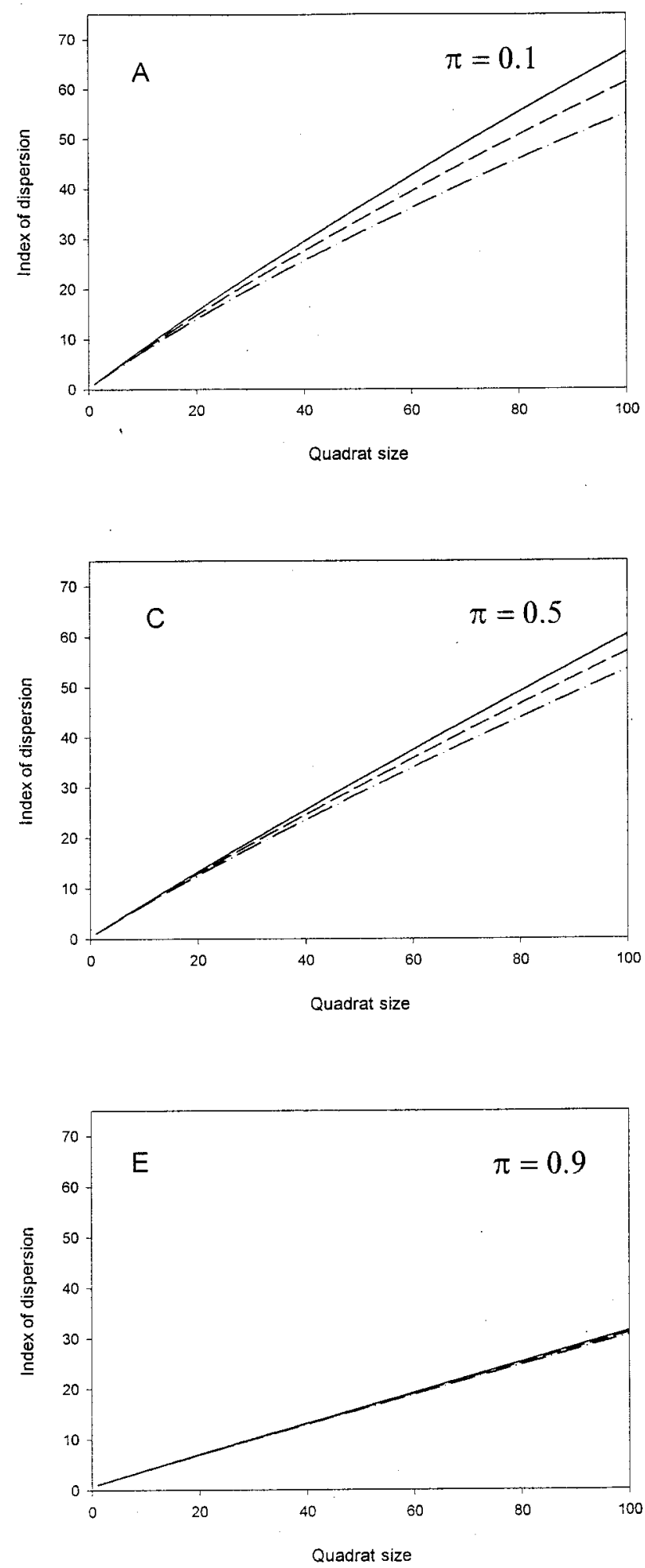

27.7 , and 24.0, respectively, and the corresponding approximate values are 19.0, 26.9, and 24.0, respectively. As we have seen earlier (Fig. 3), the approximate values underestimate the exact values of $\rho(\pi)$, and hence $I(\pi)$, when $t=0.2$ and $\pi$ is not close to 1 , particularly for quadrats with a large area and a high length/width ratio. Thus, we may expect Figure 4B, and to a less extent Figure 4D, to overstate slightly the effect of quadrat size.
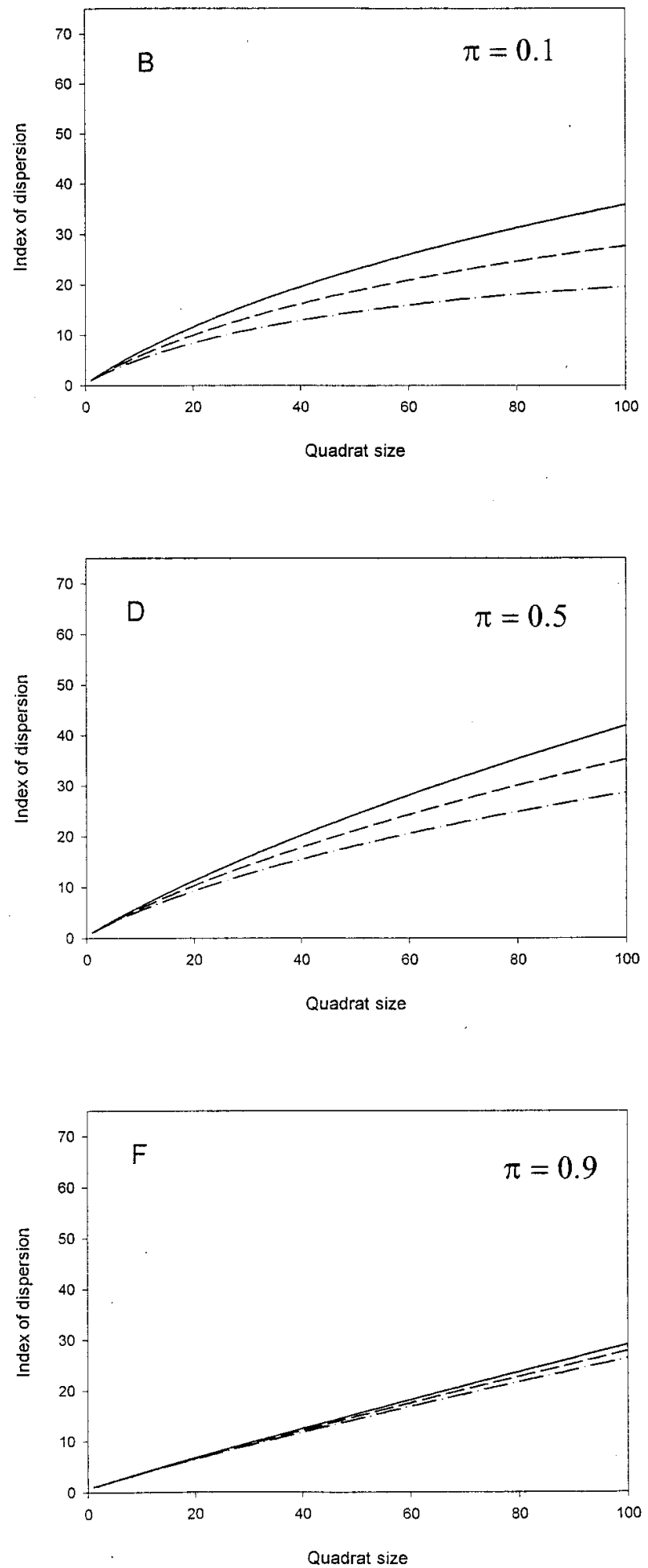

Fig. 4. Effects of quadrat size and shape on the index of dispersion based on the autocorrelation model given by equation 7 . The disease incidence, $\pi$, was 0.1 , 0.5 , or 0.9 , as indicated on the figures. The solid, dashed, and dotted-dashed lines indicate an $r / c$ ratio of 1:1, 5:1, and 10:1, respectively, in which $r$ and $c$ are the row and column dimensions of the quadrat, respectively. The parameter values in equation 7 were $\mathbf{A}, \mathbf{C}$, and $\mathbf{E}, t=0.05$; and $\mathbf{B}, \mathbf{D}$, and $\mathbf{F}, t=0.2$. $\mathbf{A}$ to $\mathbf{F}, u=$ $0.05, v=0.5$, and $S=1$. 


\section{RELATIONSHIP WITH DISTANCE CLASS ANALYSIS}

Distance class analysis has become a popular method of analyzing disease incidence data collected on a regular two-dimensional grid $(5-7,9,18,19)$. The essential idea of this approach is that the joint disease status of two plants may depend not only on the distance between them but also on their orientation relative to one another, because there are often directional effects in pathogen dispersal under field conditions. The method, therefore, considers all pairs of plants on the grid and groups them into distanceorientation classes based on the distances between plants in the horizontal and vertical directions. The total number of pairs in each distance-orientation class is counted $\left(N_{X Y}\right)$, as is the number of pairs in which both plants are infected $\left(I_{X Y}\right)$. These values are used to calculate the standardized count frequency, $\mathrm{SCF}_{X Y}=I_{X Y} / N_{X Y}(9)$. The observed standardized count frequencies are then compared with values that would be expected under a random distribution of disease incidence, though there are considerable technical difficulties with this, due to the large number of nonindependent comparisons that are made (5). The calculations can be routinely performed by the computer program 2DCLASS (19).

Previously in this paper, we have assumed that correlation depends only on distance. This has been done partly to simplify some of the mathematics and partly because, in the simulation work that we have discussed (25), dispersal was assumed to be equally likely in all directions. However, if directional effects are thought to be important, the parameter $\rho$ that is defined in equation 4 , and that has been shown to play a fundamental role in understanding aggregation, can be calculated as the average correlation over all possible distance-orientation classes. There is a direct link with the distance-orientation class approach, because the correlation within a distance-orientation class is proportional to the square root of the chi-squared statistic for comparing $I_{X Y}$ with its expected value under randomness (6).

\section{DISCUSSION}

Our aim in this paper has been to explore the relationships between various statistical methods that have been applied to disease incidence data. In particular, we have shown how the variance of the number of infected plants within a quadrat is determined by the correlations between pairs of plants within the quadrat (equations 3 and 4). This enabled us to link variance and intraclass correlation, which are not inherently spatial concepts, to spatial autocorrelation. Some simple qualitative conclusions are immediately clear from this representation. For example, since autocorrelation typically declines with increasing distance, intraclass correlation will usually decrease with increasing quadrat size. Declines in intraclass correlation with quadrat size have been observed by Madden et al. (16) and Tanne et al. (22). Strictly, these authors showed decreases in the beta-binomial parameter $\theta$ with increasing quadrat size; however, the intraclass correlation is a monotonic function of $\theta$.

More importantly, specific quantitative relationships can be investigated. We focused on the particular autocorrelation model given by $\mathrm{Xu}$ and Ridout (25), based on computer-simulated data, and showed that this resulted in a variance function that was approximated very closely by the binary form of power law, as had already been shown directly when analyzing the simulated data (25). We demonstrated also that the binary power law was reasonably symmetrical, even when the underlying autocorrelation model was highly asymmetrical. This helps to explain why the symmetrical model has often been considered adequate in practice $(10,13,15)$.

It would be straightforward to extend these investigations to other forms of autocorrelation function. Our numerical autocorrelation examples, though consistent with the simulation results by $\mathrm{Xu}$ and Ridout (25), are perhaps somewhat higher than values typically encountered in real epidemics. The overall level of correlation in our model may be reduced by reducing the scaling parameter $S$. Other features such as negative autocorrelations cannot be accommodated in our model. However, the focus of this paper is on the general method. Using this method, properties of interest such as the exact variance-mean relationship can be evaluated for any autocorrelation function.

The approximate formula derived for the mean intraclass correlation is also generally applicable, though we have illustrated its use with only one particular autocorrelation function. Although the final formula is very cumbersome, the individual components from which the formula is built up (equations 10 to 13) are less complex and are easily programmed. The approximation is particularly useful for investigating the effects of quadrat size and shape, as we have illustrated. The approximate formula produces continuous curves, as in Figure 4. For any particular quadrat shape, there are only a small number of quadrat sizes that are possible up to a given maximum. For example, if the ratio of $r / c$ is $10: 1$, the only possible quadrats with less than 100 plants are $10 \times 1,20 \times 2$, or $30 \times 3$. For none of these quadrat sizes is there a square quadrat with the same number of plants in total. Thus, in order to compare the effects of quadrat shape, as distinct from quadrat size, the smooth approximating curves in Figure 4 are very useful.

The central conclusion of this paper is that spatial autocorrelation is the most important measure of spatial structure for disease incidence data. Other authors (15) have also argued that spatial correlations at various scales provide the key to understanding spatial patterns of disease incidence. Our contribution here has been to elucidate some specific relationships mathematically. Our approach is relevant only when the spatial location of individuals is known. Indeed, our mathematical results relate to individuals arranged on a rectangular lattice, though many of the results could be generalized to allow an irregular spatial arrangement of individuals.

Once the spatial autocorrelation is known, many other measures can be derived, including the intraclass correlation (and hence the dispersion parameter of the beta-binomial distribution), the variancemean relationship, and the index of dispersion. Geostatistical methods are based on the semivariogram, which is also closely related to spatial autocorrelation (1). Spatial autocorrelation may depend only on distance, as in the main example in this paper, or it may depend also on direction. In the latter instance, spatial autocorrelation is closely linked to statistics calculated in distance class methods, as Ferrandino has pointed out (6).

Finally, although we have argued that there is a logical flow from autocorrelation through intraclass correlation to quadrat variance, we do not wish to imply that this is an appropriate sequence for data analysis. Typically, graphs of intraclass correlation against disease incidence show considerable scatter, especially for small $\pi$ values, and it is difficult to estimate parameters precisely, even when the functional form of the relationship is known. However, the parameters of the binary power law are usually much easier to estimate. These points are well illustrated by Figure 3 of Turechek and Madden (24), who suggest explanations, and are borne out in our simulation work (25). Thus, the best way to estimate the relationship of intraclass correlation to distance is indirectly, by first estimating the variance-mean relationship. In our previous simulations (25), the observed spatial autocorrelations also showed substantial scatter. Unfortunately, the variance-mean relationship does not uniquely determine the spatial autocorrelation.

\section{ACKNOWLEDGMENTS}

We thank J. Gates, University of Greenwich, United Kingdom, and R. Fisher of the Prince of Wales Hospital, Randwick, Australia, for their beneficial discussions in developing the mathematical approximation for the mean pairwise correlation. We also thank L. V. Madden and an anonymous referee for their extensive comments on an earlier draft of this paper. 


\section{LITERATURE CITED}

1. Burrough, P. A. 1987. Spatial aspects of ecological data. Pages 213-251 in: Data Analysis in Community and Landscape Ecology. R. H. G. Jongman, C. F. G. Braak, and O. F. R. van Tongeren, eds. PUDOC, Wageningen, the Netherlands.

2. Campbell, C. L., and Madden, L. V. 1990. Introduction to Plant Disease Epidemiology. John Wiley \& Sons, New York.

3. Chellemi, D. O., Rohrbach, K. G., Yost, R. S., and Sonoda, R. M. 1988. Analysis of the spatial pattern of plant pathogens and diseased plants using geostatistics. Phytopathology 78:221-226.

4. Collett, D. 1991. Modelling Binary Data. Chapman \& Hall, London.

5. Ferrandino, F. J. 1996. Two-dimensional distance class analysis of disease-incidence data: Problems and possible solutions. Phytopathology $86: 685-691$.

6. Ferrandino, F. J. 1998. Past nonrandomness and aggregation to spatial correlation: 2DCORR, a new approach for discrete data. Phytopathology 88:84-91.

7. Gottwald, T. R. 1995. Spatio-temporal analysis and isopath dynamics of citrus scab in nursery plots. Phytopathology 85:1082-1092.

8. Gottwald, T. R., Cambra, M., Moreno, P., Camarasa, E., and Piquer, J. 1996. Spatial and temporal analyses of citrus tristeza virus in eastern Spain. Phytopathology 86:45-55.

9. Gray, S. M., Moyer, J. W., and Bloomfield, P. 1986. Two-dimensional distance class model for quantitative description of virus-infected plant distribution lattices. Phytopathology 76:243-248.

10. Hughes, G., and Madden, L. V. 1992. Aggregation and incidence of disease. Plant Pathol. 41:657-660.

11. Hughes, G., and Madden, L. V. 1993. Using the beta-binomial distribution to describe aggregated patterns of disease incidence. Phytopathology 83:759-763.

12. Hughes, G., Madden, L. V., and Munkvold, G. P. 1996. Cluster sampling for disease incidence data. Phytopathology 86:132-137.

13. Hughes, G., McRoberts, N., Madden, L. V., and Nelson, S. C. 1997. Validating mathematical models of plant-disease progress in space and time. IMA (Inst. Math. Appl.) J. Math. Appl. Med. Biol. 14:85112.

14. Kendall, M. G., and Stuart, A. 1976. The Advanced Theory of Statistics. 4th ed. Charles Griffin \& Co., Ltd., High Wycombe, England.

15. Madden, L. V., and Hughes, G. 1995. Plant disease incidence: Distributions, heterogeneity, and temporal analysis. Annu. Rev. Phytopathol. 33: 529-564.

16. Madden, L. V., Nault, L. R., Murral, D. J., and Apelt, M. R. 1995. Spatial pattern analysis of the incidence of aster yellows disease in lettuce. Res. Popul. Ecol. 37:279-289.

17. Mak, T. K. 1988. Analysing intraclass correlation for dichotomous variables. Appl. Stat. 37:344-352.

18. Nelson, S. C. 1995. Spatiotemporal distance class analysis of plant disease epidemics. Phytopathology 85:37-43.

19. Nelson, S. C., Marsh, P. L., and Campbell, C. L. 1992. 2DCLASS, a two-dimensional distance class analysis software for the personal computer. Plant Dis. 76:427-432.

20. Ridout, M. S., Demétrio, C. G. B., and Firth, D. 1999. Estimating intraclass correlation for binary data. Biometrics 55:137-148.

21. Stein, A., Kocks, C. G., Zadoks, J. C., Frinking, H. D., Ruissen, M. A., and Myers, D. E. 1994. A geostatistical analysis of the spatio-temporal development of downy mildew epidemics in cabbage. Phytopathology 84:1227-1239.

22. Tanne, E., Marcus, R., Dubitzky, E., and Raccah, B. 1996. Analysis of progress and spatial pattern of corky bark in grapes. Plant Dis. 80:34-38.

23. Taylor, L. R. 1961. Aggregation, variance and the mean. Nature (Lond.) 189:732-735.

24. Turechek, W. W., and Madden, L. V. 1999. Spatial pattern analysis of strawberry leaf blight in perennial production systems. Phytopathology $89: 421-433$

25. Xu, X.-M., and Ridout, M. S. 1998. Effects of initial epidemic conditions, sporulation rate, and spore dispersal gradient on the spatio-temporal dynamics of plant disease epidemics. Phytopathology 88:1000-1012.

26. Yang, X. B. 1995. Analysis of variance-mean relationships of plant diseases. J. Phytopathol. 143:513-518. 